Published in final edited form as:

Crit Care Med. 2019 March ; 47(3): e277. doi:10.1097/CCM.0000000000003610.

\title{
Post-Intensive Care Syndrome (PICS):
}

\section{Letter to the editor}

\section{Annachiara Marra, MD, $\mathrm{PhD}^{1}$ and Nathan E. Brummel, MD, $\mathbf{M S C l}^{2,3,4,5}$}

${ }^{1}$ Department of Neurosciences, Reproductive and Odontostomatological Sciences, University of Naples, Federico II, Naples, Italy

${ }^{2}$ Division of Allergy, Pulmonary, and Critical Care Medicine, Vanderbilt University Medical Center, Nashville, Tennessee

${ }^{3}$ Critical Illness, Brain Dysfunction, and Survivorship (CIBS) Center, Vanderbilt University Medical Center, Nashville, Tennessee

${ }^{4}$ Center for Health Services Research, Department of Medicine, Vanderbilt University Medical Center, Nashville, Tennessee

${ }^{5}$ Center for Quality Aging, Vanderbilt University Medical Center, Nashville, Tennessee

\section{Keywords}

Post-Intensive Care Syndrome; Cognitive impairment; Activities of Daily Living; Depression; ICU; critical illness

We thank Dr. Ezeagu and Olanipekun for their comments (1) on our recent article in Critical Care Medicine (2). Our findings that the majority of survivors who did not have cognitive impairment or disability prior to their critical illness had at least one PICS problem a year after ICU discharge highlights the long-term effects of even a single episode of critical illness (2). We agree with the authors that subsequent episodes of critical illness are likely to result in worsening of (or developing additional new) PICS problems.

The authors hypothesize that repetitive critical illness-related psychological trauma may underlie a progressive cycle of decline in functional status. Subjective measures of health perception, such as psychological well-being, could affect how a patient recovers from critical illness and should be studied further. Nevertheless, health perception and functional status are distinct, albeit interrelated, outcome domains (3). The former is highly complex integrating not only the physiological and biological factors related to PICS problems, but individual, social and environmental factors as well. As the result, these outcomes are often not as related to objective circumstances as would be anticipated. Hence two patients with similar objective findings can vary widely in the perception of their health (e.g., one may care greatly about even minor health problems whereas another may place minimal significance on significant health problems). Outcomes such as functional status are more

Name and Address for correspondence: Nathan E. Brummel, MD MSCI, 2525 West End Avenue Suite 350, Nashville, TN 37203 , Telephone: (615) 343-3957, Fax (615) 322-1754, nathan.brummel@ vanderbilt.edu. 
closely tied to the biological and physiological disruptions present during critical illness. Thus, greater insight into the underlying mechanisms of PICS problems (and the development of effective interventions) is more likely to be gained through the study of objective measures of cognition, disability, and mental health along with associated organ system and cellular function.

We enrolled a large cohort of survivors of medical and surgical critical illness who were being treated for respiratory failure and/or shock, the majority of whom received sedatives and opiates during the course of their critical illness. We agree with the authors that shortterm morbidity can be reduced via coordinated interventions combining appropriate management of pain, agitation, delirium, and immobility (e.g., the ABCDEF bundle) (4). Nevertheless, the effects of these ICU-based interventions on longer-term PICS problems is unclear. Our finding that the majority of survivors had only a single PICS problem, suggests that no "one size fits all" PICS intervention is likely to be effective. Interventions to address problems in each of the PICS domains should be developed.

Finally, the authors propose that further study of PICS is needed in patients with less severe critical illness. The recognition that impaired physical, cognitive, and mental health function also affects those who survive hospitalizations for acute illness (5) reminds us that our efforts to understand and to improve outcomes for survivors of critical illness, should emphasize collaboration with our colleagues from hospital medicine and geriatrics whose patients face similar risks.

\section{Acknowledgments}

Dr. Brummel is supported by the National Institutes of Heath, National Institute on Aging under award K76AG054864. Dr. Brummel has received honoraria for advisory board activities from Arjo and Merck. The contents of this paper are solely the responsibility of the authors and do not necessarily represent those of the National Institutes of Health or Vanderbilt University Medical Center.

Copyright form disclosure: Dr. Brummel's institution received funding from the National Institutes of Health (NIH)/National Institute on Aging; he received funding (advisory board honoraria) from Arjo and Merck; and he received support for article research from the NIH. Dr. Marra has disclosed that she does not have any potential conflicts of interest.

\section{References}

1. Ezeagu RN, Olanipekin T: Reviewing the impact of invasive procedures on Post-Intensive Care Syndrome (PICS). Crit Care Med 2019; in press.

2. Marra A, Pandharipande PP, Girard TD, et al. Co-Occurrence of Post-Intensive Care Syndrome Problems Among 406 Survivors of Critical Illness. Crit Care Med 2018; 46(9): 1393-401. [PubMed: 29787415]

3. Brummel NE. Measuring Outcomes After Critical Illness. Crit Care Clin 2018; 34(4): 515-26. [PubMed: 30223991]

4. Pun BT, Balas MC, Barnes-Daly MA, et al. Caring for Critically Ill Patients with the ABCDEF Bundle: Results of the ICU Liberation Collaborative in Over 15,000 Adults. Crit Care Med 2018.

5. Krumholz HM. Post-hospital syndrome--an acquired, transient condition of generalized risk. N Engl J Med 2013; 368(2): 100-2. [PubMed: 23301730] 